\title{
Miradas retro-prospectivas sobre las Tecnologías Educativas
}

\section{Retro-prospective views on Educational Technologies}

\author{
Juana M. Sancho GiL ${ }^{1}$ \\ jmsancho@ub.edu \\ Cristina Alonso Cano \\ cristina.alonso@ub.edu \\ Joan-Anton Sánchez Valero \\ joananton.sanchez@ub.edu \\ Unversidad de Barcelona, España
}

\section{Resumen}

En este artículo compartimos un conjunto de reflexiones y acciones que han jalonado nuestra trayectoria académica. Un recorrido entrecruzado que, durante más de tres décadas, nos ha permitido asistir, contribuir y analizar de forma crítica los cambios que han tenido lugar en el ámbito de la Educación, en general, y el de la Tecnología Educativa (TE), en particular. Desde este panorama de partida, establecemos un diálogo con las aportaciones de Juan Manuel Escudero en torno a dos temáticas: La Tecnología Educativa más allá de las herramientas y Las Tecnologías Educativas y la formación del profesorado. El texto acaba con una reflexión prospectiva sobre el futuro-presente de la TE vinculado a las ambivalencias, los retos, y los sentidos de la educación.

\section{Palabras clave:}

Cambio educativo; formación docente; tecnologías digitales; retos educativos.

\begin{abstract}
:
In this article we share a set of reflections and actions that have oriented our academic trajectory. An intertwined itinerary that, for over three decades, has allowed us to assist, contribute and critically analyse the important changes that have taken place in the area of Education in general, and in the field of Educational Technology $(E T)$, in particular. From this starting point, we establish a dialogue with the contributions of Juan Manuel Escudero on two themes: Educational Technology beyond tools and Educational Technologies and teacher's professional development. The text ends with a prospective reflection on the future-present of ET linked to ambivalence, challenges and the meaning of education.
\end{abstract}

\section{Key words:}

Educational change; teacher's professional development; digital technologies; educational challenges

1 Dirección para correspondencia (correspondence address):

Juana M. Sancho Gil. Departamento de Didáctica y Organización Educativa. Universidad de Barcelona. Passeig Vall d'Hebron, 171. 08009 Barcelona. España. 


\section{Résumé:}

Dans cet article, nous partageons un ensemble de réflexions et d'actions qui ont marqué notre trajectoire académique. Un chemin croisé qui, pour plus de trois décennies, nous a permis d'assister, de contribuer et d'analyser de manière critique les changements importants qui ont eu lieu dans le domaine de l'éducation, en général, et plus particulièrement celui de la technologie éducative (TE). Depuis ce point de départ, nous établissons un dialogue avec les contributions de Juan Manuel Escudero autour de deux thèmes: la technologie éducative au-delà des outils et Les technologies éducatives et la formation des enseignants. Le texte se termine par une réflexion prospective sur le futur-présent de la TE liée aux ambivalences, aux défis et aux significations de l'éducation.

\section{Mots clés:}

Changement éducatif; formation des enseignants; technologies numériques; défis éducatifs.

Fecha de recepción: 8-2-18

Fecha de aceptación: 2-5-18

\section{Introducción}

La invitación a participar en este monográfico para reconocer y celebrar las aportaciones de Juan Manuel Escudero al campo de la educación, nos brinda la oportunidad de compartir un conjunto de reflexiones y acciones que han jalonado nuestra trayectoria académica. Un recorrido entrecruzado que, durante más de tres décadas, nos ha permitido asistir y contribuir a los importantes cambios que han tenido lugar en el ámbito de la Educación, en general, y el de la Tecnología Educativa, en particular. La más veterana de las autoras de este texto comenzó a vislumbrar la importancia de la Tecnología Educativa, a finales de la década de 1970 de la mano de la Psicología Ambiental, que nos hizo tomar conciencia de la importancia de la materialidad de los entornos de enseñanza en el aprendizaje (Hernández y Sancho, 1981; Sancho, 1991). Aunque en los estudios de Pedagogía la Tecnología Educativa aparecía vinculada o superpuesta a los medios audiovisuales.

En la remodelación de los planes de estudios vinculados al desarrollo de la Ley de Reforma Universitaria de 1983, en la licenciatura de Pedagogía de la Universidad de Barcelona, se incluyó una asignatura obligatoria con esta denominación. Esto nos permitió, hasta el punto que lo permitía la concepción fragmentada y compartimentalizada de los currículos -hoy todavía imperante, considerar las tres nociones de Tecnología Educativa propuestas por Davies (1996). La TE uno que pone el énfasis 
en "los soportes físicos" y en la importancia de las ayudas (medios) de enseñanza, perspectiva que conecta con uno de los primeros trabajos de Escudero (1979). La TE dos, que lo pone en los "soportes lógicos", en la importancia de las ayudas al aprendizaje, desde la perspectiva de que la tecnología del diseño del mensaje (fundamentado en la fijación de metas, análisis de tareas, principios de motivación y evaluación) subyace en el corazón del aprendizaje eficaz. Y la TE tres, que combina las dos anteriores y cuestiona el desarrollo sistemático (paso-a-paso, procedimientos rígidamente mecánicos) como la única forma de proceder, en favor de un conjunto sistémico de procedimientos (orgánicos más que mecánicos), que se centra más en los procesos que en los resultados del aprendizaje.

Esta visión nos permitió introducir en la base de la asignatura, la importancia de considerar las cuatro figuras de la Tecnología (artefactual, simbólica, organizativa y biotecnológica), propuestas desde la Filosofía de la Tecnología (Àlvarez y Méndez, 1995). Así como argumentar la imposibilidad (y la importancia) de conectar la TE con la didáctica, la organización educativa, la mejora de la educación, y el propio desarroIlo de los sistemas escolares y tecnológicos a lo largo de la historia. En definitiva, poder comenzar a hablar de Tecnologías Educativas en plural. En estas decisiones, el pensamiento y las aportaciones de Juan Manuel Escudero tuvieron un papel importante.

Sin embargo, en las diplomaturas de maestros, la prescripción, a golpe de Boletín Oficial del Estado (REAL DECRETO J440/1991, de 30 de agosto), de ofrecer una asignatura obligatoria titulada "Nuevas Tecnologías Aplicadas a la Educación", entendida como la utilización, en sus distintas aplicaciones didácticas, organizativas y administrativas, de los principales instrumentos informáticos y audiovisuales, quedó anclada en las concepciones del modo uno de TE. Todo lo anterior nos llevó a la necesidad de repensar la TE en un momento de intensificación del cambio tecnológico, económico, político, cultural y social (Sancho, 1994).

Desde este panorama de partida, en este artículo establecemos un diálogo con las aportaciones de Juan Manuel Escudero en torno a dos temáticas: La Tecnología Educativa más allá de las herramientas y las Tecnologías Educativas y la formación del profesorado. Y acabamos con una reflexión prospectiva sobre el tema. 


\section{La Tecnología Educativa más allá de las herramientas}

En la década de 1980, entran en crisis algunas de las concepciones de la $T E$, la que solo miraba al medio (enfoque instrumental); la focalizada en el sujeto (enfoque conductista); y la centrada en el diseño (enfoque sistemático). Coincidiendo con la emergencia de corrientes curriculares de naturaleza interpretativa y sociocrítica, aparecen en España las primeras voces críticas que cuestionan el discurso tecnocrático y una concepción técnico-racional del currículum y de la Tecnología Educativa (también Ilamada Tecnología de la Educación, Tecnología Educacional, Tecnología de la Instrucción o Tecnología Didáctica). Entre esas voces encontramos la del profesor Escudero (1983), quien lleva a cabo una revisión retrospectiva y crítica del trabajo realizado hasta el momento en torno a los medios. En este trabajo, Juan Manuel Escudero, cuestiona una serie de actuaciones e investigaciones centradas en el impacto, en los efectos apocalípticos y en la yuxtaposición (que no integración) de los medios en los entornos de enseñanza y aprendizaje. En este mismo artículo, ante la deficitaria contextualización practicada hasta el momento, el autor defiende la necesidad de aproximarnos y explorar los procesos de enseñanza, de redimensionar los medios y resituarlos en el currículo. "Solo una investigación comprensiva, que relacione el medio con sus contextos de uso, que siga «longitudinalmente» el devenir del medio [...], que tome en consideración la multiplicidad de dimensiones mediacionales, parecería adecuada [...] para decirnos algo provechoso sobre los materiales curriculares" (Escudero, 1983, p. 36).

Han pasado 35 años desde la escritura del trabajo recuperado en el párrafo anterior, y su relectura, una vez más, pone de manifiesto una de las reivindicaciones (no superadas) en el campo de estudio de la TE (Area, 1991; Sancho,1994; San Martín, 1995; Alonso, 1997): la necesidad de superar la dictadura de los medios (léase herramientas, tecnologías analógicas o tecnologías digitales) y los posicionamientos que encuentran en las tecnologías "la respuesta" cuando aún no se ha planteado el problema ni formulado la pregunta.

La TE, en tanto disciplina, no puede dejar de contemplar entornos de enseñanza y aprendizaje mediados por tecnologías artefactuales, simbólicas y organizativas, pero llevamos demasiado tiempo luchando contra el imperativo tecnológico de la tecnología artefactual (Àlvarez y Méndez, 1995). Una confianza ilimitada en el poder de algunas tecnologías que 
muchas profesoras y profesores del área hace tiempo que venimos cuestionando, combatiendo y denunciando.

Recuperamos ahora uno de los trabajos en los que el profesor Escudero (1995) reflexiona y establece un necesario y oportuno diálogo entre Tecnología Educativa e Innovación Educativa. En él se aborda la necesidad de construir relaciones e intersecciones entre ambas, considerando la TE como eje nuclear de las políticas, reformas y transformaciones educativas y escolares. Para el profesor Escudero, adoptar una perspectiva innovadora en educación

puede entenderse como una actitud y compromiso con el día a día de la educación con un pie en la reflexión y la crítica, en el mundo de las ideas y los valores, en la indagación y deliberación sobre el tipo de sociedad, cultura y ciudadano que queremos y debemos contribuir a formar, $y$ otro en las prácticas y relaciones que vamos promoviendo al relacionarnos con nuestros alumnos y alumnas (p.164).

Desde esta visión, "la tecnología educativa merece ser entendida como una mirada y un conjunto de procesos y procedimientos, no sólo de aparatos" (p.162). Partiendo de este posicionamiento el autor argumenta que las políticas de cambio e innovación en el mundo educativo no pueden dar la espada al nuevo mundo de la información y la tecnología, sin dejar de considerar y sopesar "cuáles pueden y deben ser los criterios, principios y valores que hayan de orientar los por qués, para qués y cómos de la presencia y tratamiento educativo de la tecnología" (p. 161).

El mismo año en el que se publicó el trabajo al que nos hemos referido en el párrafo anterior, el profesor Escudero participó como ponente en las III JUTE (Jornadas Universitarias de Tecnología Educativa), celebradas en Barcelona en el año 1995, y casi 20 años más tarde vuelve a intervenir, también como conferenciante invitado, en las XXII JUTE que tuvieron lugar en Toledo en el año 2014. En ambas intervenciones (Escudero, 1997 y 2014), sigue explorando y reflexionando en torno a la Tecnología Educativa desde la perspectiva de la innovación y la mejora de la educación. En el cierre de su intervención en Barcelona, Juan Manuel Escudero, haciendo referencia a las macropolíticas de reforma, reclamaba repensar la Tecnología Educativa no dejándose 
embaucar fácilmente por los cantos de sirena de los nuevos reformadores, sino situar la tecnología y su presencia social, cultural y educativa en las coordenadas de una discusión más amplia sobre qué tipo de sociedad, qué tipo de cultura, qué tipo de ciudadano, y así, qué escuela y qué educación (Escudero, 1997, p.47).

Casi dos décadas más tarde, el final de la ponencia del Dr. Escudero en las JUTE de Toledo, no difiere demasiado de la del año 1995 en Barcelona:

En resumidas cuentas, el horizonte de las innovaciones, las mejoras y las transformaciones por las que hay que pelear apuntan a que sean, en algunos sentidos, efectivamente profundas e integrales, en otros y simultáneamente, a que sean concertadas, comprometidas y llevadas a cabo por agentes, instituciones y servicios múltiples, debidamente vertebrados en torno a proyectos sólidos, consistentes y sostenidos en el tiempo (Escudero, 2014, p. 36-37).

Quienes firmamos este trabajo, nos sentimos muy próximos al discurso y a la obra del profesor Escudero en su concepción de una Tecnología Educativa más allá de las herramientas. En todo este tiempo han proliferado y siguen proliferando grandes discursos, acciones políticas y prácticas entusiastas en torno a las posibilidades de las tecnologías (ahora digitales) en la educación, pero son muchas las evidencias que ponen de manifiesto las promesas incumplidas por las tecnologías (Sancho, Bosco, Ornellas, Sánchez y Alonso, 2008). Realmente resulta agotador, como decíamos al inicio de este apartado, cuestionar, combatir y denunciar una y otra vez el discurso de la "Tecnología Educativa fácil", la que no es capaz de ir "más allá de las herramientas", la que desdibuja (hasta diluir) el discurso pedagógico en el tecnológico. Una Tecnología Educativa reduccionista que es incapaz de superar unos escenarios y unas prácticas educativas mediadas, en estos momentos, por las TIC que no se cuestionan. Pero justamente no son interpeladas porque el mero hecho de utilizar determinadas herramientas digitales es un indicador de modernidad, de estar al día. Como decíamos en otro lugar (Alonso, 2011 y 2012), en definitiva, a menudo, no se trata mas que de unas prácticas continuistas, asociadas a propuestas pedagógicas que contribuyen a anclar y afianzar la gramática de la escuela (Tyack y Tobin, 1994). Sin embargo, como muestra la investigación, la presencia de artefactos digitales no siempre 
garantiza que los estudiantes se relacionen con ellos de forma crítica, reflexiva y creativa (Sancho y Alonso, 2012).

\section{Tecnologías educativas y la formación del profesorado}

\section{La formación inicial del profesorado en tecnologías educativas}

Como hemos señalado, la primera asignatura obligatoria vinculada con las tecnologías educativas en la formación inicial del profesorado aparece en las directrices de los planes de estudios de maestro de 1991 como materia troncal": "Nuevas tecnologías aplicadas a la educación. Recursos didácticos y nuevas tecnologías: utilización en sus distintas aplicaciones didácticas, organizativas y administrativas. Utilización de los principales instrumentos informáticos y audiovisuales", asignándole 4 créditos. La inclusión de la palabra "utilización" al inicio de las dos frases descriptivas de la materia daba perfecta cuenta del carácter aplicado que se le quería dar a las tecnologías educativas en la formación de maestros, obviando la necesaria reflexión y visión crítica sobre dichas tecnologías como reclamaba Cabero (1992): “... su formación es una condición imprescindible en tanto que oportunidad para justificar, comprender y decidir de una manera más reflexiva y crítica qué hacer, cómo, por qué y para qué con el ordenador" (p. 121).

En la línea reclamada por Cabero, Estebanell y Ferrés (1993) analizaron la asignatura de Nuevas Tecnologías Aplicadas a la Educación (NTAE) considerando que además de formar a los futuros maestros en el conocimiento de las herramientas, era necesario capacitarlos sobre sus implicaciones educativas. Asimismo, creían que dichas tecnologías tenían que transversalizarse, es decir, referenciarse en todas las áreas curriculares. En consonancia con las consideraciones anteriores, la mayoría del profesorado de las diplomaturas de maestro fue más allá de los planteamientos asignados a la asignatura NTAE, como revelaba un análisis de los programas (Quintana y Vivancos, 2000) que destacaba que dichos programas compartían objetivos relativos a la reflexión crítica sobre el papel que tenían los medios de comunicación en la sociedad, los retos

2 Real Decreto 1440/1991, de 30 de agosto. Publicado en el BOE núm. 244, de 11 de octubre de 1991. Disponible en https://www.boe.es/buscar/doc.php?id=BOEA-1991-24768 
que representaban para las escuelas, los enfoques teóricos relacionados con las TIC, etc.

A diferencia de las directrices de los planes de estudio de 1991, la actual planificación de los títulos de los grados de maestro, de $2007^{3}$, no concreta ningún módulo específico de tecnologías educativas, sino que establece unas competencias generales que se abordan en diferentes módulos. En el grado de Educación Infantil: "Conocer las implicaciones educativas de las tecnologías de la información y la comunicación y, en particular, de la televisión en la primera infancia" y en Educación Primaria "Conocer y aplicar en las aulas las tecnologías de la información y de la comunicación. Discernir selectivamente la información audiovisual que contribuya a los aprendizajes, a la formación cívica y a la riqueza cultural".

En relación con dicha planificación, Ornellas y Sánchez (2008), objetaban que la opción por incorporar la formación relacionada con las tecnologías digitales en los distintos módulos podría ayudar a los estudiantes a tener una visión más transversal de éstas, pero podría comportar una menor competencia digital docente de los futuros graduados. Dicha objeción resultó premonitoria, ya que, desde la implementación de los grados de maestro, se ha agudizado la preocupación respecto al hecho que los estudiantes de maestro no reciban la adecuada formación en relación con las tecnologías digitales. Por un lado, nos encontramos que dichas tecnologías tienen un tratamiento muy diferente en los planes de estudio de las universidades; y por otro que la opción por la transversalidad ha hecho que el profesorado universitario les esté dando un tratamiento desigual de acuerdo con sus concepciones sobre las tecnologías (Sancho, Bosco, Alonso y Sánchez, 2015).

Asimismo, las actuales directrices de los grados de maestro parten de una visión básicamente instrumental de las tecnologías digitales, desaprovechando el potencial de éstas como facilitadoras de procesos de innovación educativa, sobre todo en relación con el trabajo colaborativo y la construcción de conocimiento. También sorprende que las competencias vinculadas a la didáctica de la Lengua se circunscriban al ámbito oral y escrito, cuando en las necesidades formativas en nuestra socie-

3 Orden ECl/3854/2007, de 27 de diciembre. Publicada en el BOE núm. 312, de 29 de diciembre de 2007. Disponible en https://www.boe.es/diario_boe/txt.php?id=BOEA-2007-22446. Orden ECI/3857/2007, de 27 de diciembre. Publicada en el BOE, núm. 312, de 29 de diciembre de 2007. Disponible en https://www.boe.es/diario_ boe/txt.php?id=BOE-A-2007-22449 
dad hacen necesaria la competencia en los nuevos alfabetismos (Sancho, 2006).

En el caso de la formación del profesorado de secundaria la situación es igualmente preocupante. En el antiguo Curso de Aptitud Pedagógica (CAP), de organización compacta y de corta duración, era difícil encontrar asignaturas vinculadas a la formación pedagógica en el ámbito de las tecnologías digitales (Ornellas y Sánchez, 2008). En 2007 se establece la creación de un máster que será requisito para el ejercicio de las profesiones de Profesor de Secundaria Obligatoria, Bachillerato, Formación Profesional y Enseñanza de Idiomas ${ }^{4}$.

El cambio de modelo formativo del profesor de secundaria, si bien es una mejora respecto a CAP, podía haber sido una oportunidad para incorporar las tecnologías digitales en su formación inicial, pero éstas no son recogidas como materia en la planificación del máster de secundaria y únicamente se hace referencia a ellas en las competencias genéricas: "Buscar, obtener, procesar y comunicar información (oral, impresa, audiovisual, digital o multimedia), transformarla en conocimiento y aplicarla en los procesos de enseñanza y aprendizaje en las materias propias de la especialización cursada" y en el módulo de aprendizaje y enseñanza de las materias: "Integrar la formación en comunicación audiovisual y multimedia en el proceso de enseñanza-aprendizaje".

Por lo que respecta a los planes de estudio del máster de secundaria, un análisis de éstos en diferentes universidades (Sancho, Bosco, Alonso y Sánchez, 2015) revela que los contenidos o referencias a las tecnologías educativas son meramente anecdóticos e insuficientes para formar a unos profesionales capaces de comprender, acompañar y dar respuestas a las necesidades de los jóvenes de los centros de secundaria.

La definición de la competencia digital docente por parte del Ministerio de Educación, Cultura y Deporte (INTEF, 2017) y de algunas comunidades autónomas como Cataluña ${ }^{5}$ que se prevé sea un requisito obligatorio para el acceso al ejercicio de la docencia, es un atisbo de esperanza para que se modifiquen los planes de estudio de los grados de maestro incorporando dichas competencias. En esta línea el proyecto

4 Orden $\mathrm{ECl} / 3858 / 2007$, de 27 de diciembre. Publicada en el BOE núm. 312 de 27 de diciembre de 2007. Disponible en https://www.boe.es/diario_boe/txt.php?id=BOEA-2007-22450

5 Resolución ENS/1356/2016, de 23 de mayo. Publicada en el DOGC Núm. 7133. 2.6.2016. Recuperado de: http://portaldogc.gencat.cat/utilsEADOP/ PDF/7133/1500244.pdf 
"Proposta interuniversitària de formació inicial de mestres en tecnologies digitals" ${ }^{\prime}$ está elaborando una propuesta que contempla tres acciones: 1) un módulo obligatorio de formación específica; 2) una distribución de los descriptores de la CDD en los módulos de los planes de estudios; y 3) una mención en Tecnologías Digitales.

\section{La formación permanente del profesorado en tecnologías educativas.}

Los primeros planes de formación en informática educativa para el profesorado aparecen a principios de la década de 1980, como las formaciones intensivas, extensivas y modulares organizadas en Cataluña por el "Centre de Recursos d'Informàtica Educativa i Professional" que desarroIla sus actividades entre 1982 y 1986 (Bertran, Berga, Butzbach, Orgué, et al., 1985; Ruiz, 2007). A mediados de la década se van creando nuevos programas que daban cobertura y legitimación oficial a las primeras experiencias pioneras (Area, 2006) e incluían planes de formación en informática del profesorado. En 1985 el Ministerio de Educación y Ciencia crea el Proyecto Atenea, evaluado por un equipo dirigido por el profesor Juan Manuel Escudero (PNTIC, 1989), y en 1986 se inicia en Cataluña el Proyecto de Informática Educativa (Ruiz, 2007).

El proyecto Atenea fue un hito en la informática educativa en el ámbito del territorio vinculado con el Ministerio de Educación y Ciencia: "El programa hizo unos esfuerzos notables tanto por lo que hace al equipamiento de los centros y los equipos, como también en la dedicación de unos tiempos considerables al mismo tema de la formación de monitores y profesores" (Cabero, 1993, p. 130). A pesar de todo, el modelo formativo de dicho programa primó unos contenidos centrados en el ordenador y los programas informáticos. Según las encuestas realizadas a profesores (PNTIC, 1989), la incidencia de la integración de las tecnologías en su formación había sido escasa, sobre todo en relación con el trabajo en grupo, la compartición de experiencias y la evaluación. En definitiva, consideraban que su formación "ha sido más extensa que no intensa, poco práctica y muy insuficiente por lo que hace a su contenido "pedagógico»" (Cabero, 1993, p. 125).

A pesar de las avaluaciones de la formación en tecnologías digitales, como la dirigida por Cabero, la concepción de la formación basada en

6 Proposta interuniversitària de formació inicial de mestres en tecnologies digitals (2015 ARMIF 00027). Página web: http://fimted.cat/ 
el artefacto como talismán que producirá de manera automática un uso pedagógico innovador entre los docentes impregna, hasta principios del siglo XXI, la mayoría de los programas de formación del Ministerio de Educación y de las consejerías de las Comunidades Autónomas como en el caso de Cataluña (Ornellas y Sánchez, 2008). El nuevo siglo marca un punto de inflexión en algunos programas de formación institucionales, como en el caso de el plan de formación permanente del profesorado 2005-2010 en Cataluña (Ornellas, Sánchez, Fraga y Domingo, 2015), caracterizado por priorizar los procesos de enseñanza y aprendizaje, y enfatizar las posibilidades educativas que ofrecen las tecnologías digitales. Asimismo, el aumento de los asesoramientos a centros en detrimento de los cursos genéricos supone un avance en la contextualización de dichas tecnologías en diversos modos de entender el currículo y diferentes modelos pedagógicos. A pesar de dicha inflexión, en el proyecto "Políticas y prácticas en torno a las TIC en la enseñanza obligatoria: Implicaciones para la innovación y la mejora" (Sancho y Alonso, 2012) se llegó a la conclusión que las nuevas políticas resonaban de manera muy tímida en los centros educativos, por lo que no podemos poner toda la fuerza de la transformación de la escuela solo en las políticas educativas (Ornellas, Sánchez, Alonso y Moltó; 2009).

La aparición de los programas 1x1, tales como Escuela 2.0 (Area et al., 2014), suponen un paso atrás en la priorización de los aspectos pedagógicos de las tecnologías digitales, un retorno a la máquina que no dejará espacio para la necesaria reflexión sobre dichas tecnologías que, como se ha comentado, reclamaba Cabero a principios de los años noventa. Los programas $1 \times 1$ no se renovarán, fruto o excusa del escenario de crisis económica que está comportando una reducción drástica de los recursos destinados a la formación del profesorado de la que todavía no nos hemos recuperado.

Como en la formación inicial, la identificación de la competencia digital docente y la necesidad de acreditación del profesorado en este campo se vislumbra como una esperanza que posibilite que la competencia digital metodológica sea el eje de la futura formación del profesorado. Esperemos, una vez más, que la historia no se repita. 


\section{El futuro-presente de la TE: ambivalencias, retos, sentidos de la educación}

Desde la publicación de "Tecnología educativa: diseño de material escrito para la enseñanza de conceptos" (Escudero, 1979) y de "Para una tecnología educativa" (Sancho, 1994) muchos aspectos del mundo se han transformado de manera profunda e irreversible. Mientras que otros (las relaciones de poder, la redistribución de la riqueza, la necesidad de conciencia de sí y el tiempo y condiciones, que requiere el aprendizaje con sentido...) permanecen igual -o para algunos peor. Y como argumentaba Pareto (1966), es ese contraste entre lo que cambia y lo que persiste, o en algunos casos empeora, lo que provoca las mayores turbulencias. Por lo que las personas interesadas y conscientes de la importancia de la educación, afrontamos la necesidad de considerar esas turbulencias. De ahí que en este último apartado nos planteemos una mirada prospectiva, desde la consideración de las causas tecnológicas, científicas, económicas y sociales que aceleran la evolución del mundo actual, y la previsión de las situaciones que podrían derivarse de sus influencias.

Los clarividentes argumentos de Harold Innis (1894-1952), sobre los tres efectos de las nuevas tecnologías de la información y la comunicación, parecen más vigentes que nunca. Para este autor, estas tecnologías alteran la estructura de nuestros intereses (las cosas en las que pensamos). Cambian y amplían el carácter de los símbolos (las cosas con las que pensamos). Y modifican la naturaleza de la comunidad (el área en la que se desarrolla el pensamiento) (en Tedesco, 1995). De lo que parece no caber duda es que las tecnologías digitales no solo han modificado de manera profunda y compleja la forma de producir, almacenar, acceder y valorar la información. Para autores como Carr (2011) y Tweenge (2107) también han transformado, no solo nuestra forma de aprender, sino también de relacionarnos y situarnos en el mundo que nos rodea.

Esta situación, unida a las tendencias políticas y económicas que caracterizan la segunda década del siglo XXI, plantea una serie de cuestiones que los sistemas educativos no pueden dejar de encarar.

En primer lugar, nos referimos, una vez más, a cómo persiste y aumenta la idea de que solo los desarrollos digitales son tecnología. Algo que se desprende de la pregunta de la periodista Pilar Álvarez (2017) a Richard Gerver, asesor del Gobierno británico: “¿Puede la tecnología sustituir a los libros de texto?". Como si los libros no fuesen una 
tecnología de la información sino algo natural. O en de los títulos de libros como Distrusting Educational Technology: Critical Questions for Changing Times o Is Technology Good for Education? (Swelyn, 2013; 2016). Esta superposición podría llevar a desconsiderar las diferentes dimensiones de la tecnología, en general, y de la Tecnología Educativa en particular. A olvidarnos de que como argumentaba Mecklenburger (1990, p. 106), "la escuela es una 'tecnología' de la educación, del mismo modo que los coches son una 'tecnología' del transporte". De que las tecnologías peligrosas son aquellas que "han desaparecido", aquellas que "han llegado a formar parte del entramado de la vida cotidiana hasta que no logramos distinguirlas de ella" (Weiser, 1991, p. 94). Lo que Ileva a seguir pensando que la "tecnología" es un destino inevitable y no como argumenta Feenberg (1991), un parlamento de las cosas en el que alternativas civilizatorias se debaten y deciden.

En segundo, a la importancia de no dejar la Tecnología Educativa en manos de las grandes empresas tecnológicas, un tema que resultará cada vez más difícil. Tradicionalmente han sido los arquitectos, los diseñadores de muebles escolares, las editoriales de libros de texto y materiales de enseñanza los que han orientado el sentido de la Tecnología Educativa, de las "formas de hacer" la educación (Sancho, 1996). Del mismo modo que el desarrollo de los distintos dispositivos tecnológicos que se han ido intentado introducir en los sistemas de enseñanza siempre ha tenido lugar fuera del campo de la educación, casi siempre, con la finalidad de resolver problemas específicos del ejército (Noble, 1991), la economía, la investigación, o simplemente para aumentar el consumo.

De ahí que en un momento en el que las grandes corporaciones tecnológicas han (re)descubierto los enormes beneficios que les puede reportar la educación (Sancho, 2017), resulte crucial explorar críticamente las visiones optimistas surgidas en torno al uso de la tecnología digital en la educación. Evidenciar cómo formas aparentemente neutras de tecnología educativa han servido realmente para alinear la oferta y las prácticas educativas con los valores neoliberales, erosionando la naturaleza de la educación como bien público y moviéndola hacia las tendencias individualistas del capitalismo del siglo XXI (Swelyn, 2013). Así como resulta perentorio cuestionar la premisa de que la digitalización de la educación está "sin duda" siendo positiva y ofrecer contrapuntos críticos que desafíen algunas de las tesis centrales de esta visión. Pero, sobre todo, como viene sucediendo con todos los desarrollos tecnológicos a 
través de la historia, parece decisivo analizar lo que se va perdiendo a medida que estas tecnologías ocupan un papel cada vez más fundamental en los sistemas educativos. Cuestionar los valores, agendas e intereses que más se benefician del auge de la educación digital. Y considerar enfoques alternativos que podrían ser capaces de rescatar y tal vez revitalizar los ideales de la educación pública, sin negar por ello las posibilidades de la tecnología digital (Swelyn, 2016). Esta visión crítica, basada en evidencias y situada en una perspectiva educativa orientada hacia el reequilibrio de la desigualdad y la asunción de responsabilidades en la defensa de aquello que nos es común (Garcés, 2013), precisa de un profundo conocimiento de la sociedad actual, de su influencia en nuestros comportamientos y de las características, fortalezas y peligros de la tecnología digital.

En tercer lugar, nos referimos a la creciente necesidad de que los responsables de los sistemas educativos (políticos, investigadores, formadores de docentes, profesorado, personal de asesoramiento y apoyo y las propias familias) entiendan cómo aprenden los niños y jóvenes hoy. El aprendizaje no es algo natural (Biesta, 2014).

El aprendizaje es un fenómeno que involucra a personas reales que viven en contextos sociales reales y complejos de los que no se pueden abstraer de manera significativa. [...] los aprendices están contextualizados. Pertenecen a un género, tienen una orientación sexual, un nivel socioeconómico, son parte de una etnia, de una cultura de origen; tienen intereses -y cosas que les aburren; pueden o no haber desayunado; y viven en barrios con o sin frecuente violencia armada o terremotos, les atrae (o se enfrentan con) la personalidad de sus docentes... (Phillips, 2014, p. 10).

Y no solo se precisa tener en cuenta esto sino dónde, qué, con qué y con quién aprenden, porque hoy el aprendizaje está más distribuido que nunca y las tecnologías digitales representan, en sentido positivo o negativo, un papel fundamental en los procesos de aprendizaje de las personas (Hernández-Hernández, 2017; Twenge, 2017). En un sistema educativo que busca el bien común y no las ingentes ganancias de algunas empresas, conocer y entender este contexto; indagar sobre los beneficios y los perjuicios que tiene para el aprendizaje y la capacidad de ser del alumnado; y diseñar entornos de aprendizaje con las tecnologías y las culturas más adecuadas para lograr los fines de la educación 
propuestos, constituyen retos fundamentales para la educación actual. Porque si no los afrontamos los educadores, lo harán las empresas, ya lo están haciendo.

En cuarto y último lugar, consideramos la proliferación apabullante de dispositivos tecnológicos digitales. Durante siglos se vio al desarrollo tecnológico como una forma de resolver los problemas de la humanidad, aunque cada solución trajese o evidenciase otros problemas (Mumford, 1982). Cuando a comienzos de la década de 1980, uno de nosotros participaba en la implementación del primer programa de informática educativa desarrollado en nuestro país (Sancho y Butzbach, 1985), un colega inglés que impartía un seminario afirmó que "los ordenadores eran soluciones en busca de problemas". Que en aquellos momentos los educadores nos estábamos rompiendo la cabeza pensando en cómo aplicarlos a la educación. Hoy las cosas han cambiado profundamente y parece que lo harán más en el futuro próximo y las tecnologías digitales no solo resuelven, sino que inventan y crean problemas. $Y$ en el campo de la educación se están convirtiendo en un gran problema (Carr, 2011; Jubany, 2017; Twenge, 2017). Primero, por los efectos casi hipnóticos y a la vez de dispersión, sensación falsa de conexión, desvalorización de la información debido a su propio exceso, impresión ilusoria de autoría, dependencia de la mirada de los otros, etc. Segundo porque

El conocimiento es reflexión sobre la información, es capacidad de discernimiento y de discriminación respecto a la información que se tiene, es capacidad de jerarquizar, de ordenar, de maximizar, etc., la información que se recibe. [...]. Es decir, todo es información menos el conocimiento que nos permite aprovechar la información (Savater, 1998, p. 2-3).

Por lo que la elaboración de conocimiento necesita, además de atención -un bien escaso, entornos de reflexión y de acción que permitan desarrollar marcos de pensamiento que favorezcan el sentido.

De ahí que el principal problema para abordar este problema -de ahí el reto y la necesidad de ampliar la perspectiva de la TE, tenga al menos tres vertientes. Una, que los propios docentes, los formadores y los responsables de las políticas educativas, desconocemos los entresijos de unas aplicaciones cada vez más opacas, que además de manejar una enorme cantidad de datos sobre millones y millones de personas, generan adicción (García, 2018). Dos, la falta de medios y formación para 
el profesorado, los centros y los sistemas educativos como un todo para rediseñar los espacios de enseñanza y aprendizaje. Y tres, la limitación de los recursos y el tiempo necesarios para poder entender el sentido del impacto creciente de las tecnologías digitales en el modo de aprender de los seres humanos.

\section{Conclusiones}

El articulado de este texto, en el que las aportaciones del profesor e investigador Juan Manuel Escudero han servido de hilo conductor, más que llevarnos a conclusiones nos lleva a señalar los retos y desafíos que tenemos pendientes.

Para los interesados en ofrecer una educación con sentido y a la altura de la complejidad de nuestro tiempo, el primero se refiere a la importancia y absoluta necesidad, de desarrollar y poner en práctica una visión amplia, profunda y transdisciplinar de la Tecnología Educativa, o mejor, de las Tecnologías Educativas. Una perspectiva que contemple el entramado artefactual, simbólico, organizativo -e incluso biotecnológico, que componen las instituciones educativas y tenga en cuenta toda la investigación relativa a los componentes, influencias, factores y condiciones que facilitan (o dificultan) el aprender.

Mientras que el segundo, tiene que ver con importancia promover una perspectiva educativa crítica en relación al papel social y económico de las tecnologías digitales. Tema que Juan Manuel Escudero ya abordó en el I Congreso Europeo sobre Tecnología de la Información en la Educación: Una visión crítica.

The growing technologization of the social life and the rates of production are provoking important changes, but it is not obvious that those changes are very far from transforming seriously all the cultural and economic inequalities which dominate in a society like ours. All the changes and progresses associated with the terminological development not only don't touch the social, dominant, hierarchical and powerful relationships but, in a delicate way, tend to perpetuate and to legitimize them as a new kind of power, control and domination. In this way, the blindness due to the presence of several technological, social, labour and economic changes, tends to hide what their real character is, what the purposes of their interests are, and what is the kind 
of society and relationships which is being built and supported by our dominant model of progress (Escudero, 1992, p. 57).

Afrontar este tipo de problemas sociales y educativos requiere ir más allá de las visiones centradas en los dispositivos tecnológicos digitales, que no tienen en cuenta ni los contextos e intereses que llevan a su producción, ni las consecuencias centrales y colaterales derivadas de su uso.

\section{Referencias bibliográficas}

Alonso, C. (1997). La tecnología Educativa en los noventa: inquietud en la mirada. En C. Alonso (Coord.), La Tecnología Educativa a finales del siglo XX: concepciones, conexiones y límites con otras disciplinas (pp.103-113). Barcelona: Eumo Gràfic. III

Alonso, C. (2011). Las TIC en Cataluña, Cuadernos de Pedagogía, 418, 40-43.

Alonso, C. (2012). La evolución de las políticas de uso de las TIC en la educación en Cataluña. En J.M. Sancho y C. Alonso (Comp), La fugacidad de las políticas, la inercia de las prácticas (pp.21-33). Barcelona: Octaedro.

Àlvarez, A. y Méndez, R. (1995). Cultura tecnológica y Educación. En J.M. Sancho y L. M. Millán (Comp.), Hoy ya es mañana. Tecnologías y Educación: un diálogo necesario (pp. 21-36). Sevilla: Publicaciones M.C.E.P.

Area, M. (1991). La Tecnología Educativa en la Actualidad: las evidencias de una crisis, Qurriculum, 3, 3-18.

Area, M. (2006). Veinte años de políticas institucionales para incorporar las TIC al sistema escolar. En J. M. Sancho (coord): Tecnologías para transformar la educación (pp. 199-232). Madrid: Universidad Internacional de Andalucía y AKAL.

Area, M, Alonso, C., Correa, J.M., Moral, M.E. de Pablos, J., Paredes, J. y Valverde, J. (2014). Las políticas educativas TIC en España después del Programa Escuela 2.0: las tendencias que emergen. RELATEC: Revista Latinoamericana de Tecnología Educativa, 13(2), 11-34.

Bertran, M., Berga, J., Butzbach, M., Orgué, J., Roig, P., Sales, P. y Sancho, J. M. (1985). La informatització de l'ensenyament professional a Catalunya: un model integrat. Novàtica, 61, 4-31.

Biesta, G. (2014). Beautiful Risk of Education. London, UK: Paradigm Publishers.

Carr, N. (2011). The Shallows: What the Internet Is Doing to Our Brains. New York/London: W. W. Norton \& Company.

Davies, I.K. (1996). Educational technology: archetypes, paradigms and models. En D. P. Ely, y T. Plomp (Eds.), Classic Writings on Instructional Technology (pp. 15-29). Englewood, CO: Libraries Unlimited, Inc.

Escudero, J. M. (1979). Tecnología educativa: diseño de material escrito para la enseñanza de conceptos. Valencia: Universitat de València.

Escudero, J.M. (1983). Nuevas reflexiones en torno a los medios para la enseñanza. Revista de investigación educativa, RIE, 1(1), 19-44. 
Escudero, J. M. (1992). Integration of the New Inform.ation Technologies in Schools. European Conference about Information Technology in Education: a critical insight. Lectures (pp. 53-74). Barcelona: Universidad de Barcelona.

Escudero, J. M. (1995). Tecnología e Innovación Educativa. Bordón. Revista de Pedagogía, 47(2), 161-175.

Escudero, J.M. (1997). Tecnología Educativa: algunas reflexiones desde la perspectiva de la innovación y la mejora de la educación. En C. Alonso (Coord.), Op. Cit. (pp. 38-48).

Escudero, J.M. (2014). Contexto, contenidos y procesos de innovación educativa: ¿el dónde y el cómo de la tecnología educativa? Docencia e Investigación: revista de la Escuela Universitaria de Magisterio de Toledo, 39(24), 13-36.

Estebanell, M. y Ferrés, F. (1993). Las nuevas tecnologías aplicadas a la educación como materia en los planes de estudio. En J. de Pablos (coord.), La tecnología educativa en España: actas de las I Jornadas Universitarias de Tecnología Educativa (pp. 129136). Sevilla: Universidad de Sevilla.

Feenberg, A. (1991). The Critical Theory of Technology. New York: Oxford University Press. Garcés, M. (2013). El compromís. Barcelona: CCCB.

García, J. (2 de febrero de 2018). Ex trabajadores de Facebook y Google alertan sobre los enormes peligros de usar sus apps. Tecnología. Recuperado de https://andro4all. com/2018/02/ex-trabajadores-facebook-google-peligro

Hernández-Hernández, F. (Coord.). (2017). ¡Y luego dicen que la escuela pública no funciona! Investigar con los jóvenes sobre cómo transitan y aprenden dentro y fuera de los centros de Secundaria. Barcelona: Octaedro.

Hernández, F. y Sancho, J. M. (1981). Interacción ambiental en el parvulario. Barcelona: Publicaciones del ICE de la Universidad de Barcelona.

INTEF (2017). Marco Común de Competencia Digital Docente. Recuperado de: http:// educalab.es/documents/10180/12809/MarcoComunCompeDigiDoceV2.pdf

Jubany, J. (2017). ¿Hiperconectados? Educarnos en un mundo digital. Barcelona: Lecto.

Mecklenburger, J. A. (1990). Educational Technology Is Not Enough. Phi Delta Kappan, 72(2), 104-108.

Mumford, L. (1982). Técnica y civilización. Madrid: Alianza.

Noble, D. N. (1991). The Classroom Arsenal: Military research, Information Technology, and Public Education. The Falmer Press.

Ornellas, A. y Sánchez, J. A. (2008). Una mirada hacia la formación del profesorado en Tecnologías de la Información y la Comunicación en Cataluña. Diálogos. Educación y formación de personas adultas, 55(II), 49-62.

Ornellas, A., Sánchez, J. A., Fraga, L. y Domingo, L. (2015). Políticas y prácticas en la formación permanente del profesorado en TIC en Cataluña. Revista Electrónica Interuniversitaria de Formación del Profesorado, 18(3), 83-96. doi: 10.6018/reifop.18.3.190271

Ornellas, A.; Sánchez, J. A.; Alonso, K. y Moltó, O. (2009). Two decades of ICT Policy in Education. Changing discourses. Changing practices? En A. Méndez, A. Solano, J. Mesa y J. A. Mesa (eds.) Research, reflections and innovations in integrating ICT in education (pp. 154-157). Badajoz: Formatex. 
Phillips, D. C. (2014). Research in the Hard Sciences, and in Very Hard "Softer" Domains. Educational Researcher, 43(1), 9-11. DOI: 10.3102/0013189X13520293, 2014.

PNTIC (1989). Informe de progreso. Fase exploratoria (Proyecto Atenea). Madrid: Ministerio de Educación y Ciencia. Recuperado de https://sede.educacion.gob.es/ publiventa/d/20927/19/0

Quintana, J. y Vivancos, J. (2000). Teacher Training and Information and Communication Technologies in Spain. Recuperado de https://web.archive.org/web/20070804180112/ http://settt.europole.u-nancy.fr:80/anglais/ntic1.asp

Ruiz, F. (2007) Vint-i-cinc anys de polítiques d'integració de les TIC als centres docents de Catalunya. En J. M. Mominó y C. Sigalés (dir.) L'escola a la societat xarxa: Internet a l'educació primària i secundària (pp. 29-180). UOC y IN3. Recuperado de http://www.uoc.edu/in3/pic/cat/pdf/pic_escola_capitol2.pdf

San Martín, A. (1995). De la "miseria" del método a la "grandeza" de las tecnologías. En J.M. Sancho y L. M. Millán (Comp.), Op. Cit. (pp. 75-93).

Sancho, J. M. (1991). El entorno físico y simbólico de la enseñanza. Cuadernos de Pedagogía, 192 73-77.

Sancho, J. M. (1994a). La tecnología: un modo de cambiar el mundo cargado de ambivalencia. En J. M. Sancho (Coord.), Para una Tecnología Educativa (pp. 13-38). Barcelona: Horsori.

Sancho, J. M. (Coord.) (1994b). Para una tecnología educativa. Barcelona: Horsori.

Sancho, J. M. (1996). Las tecnologías educativas como "formas de hacer" la educación. XI Congreso Nacional de Pedagogía (pp. 145-164). Madrid: Sociedad Española de Pedagogía.

Sancho, J. M. (2006). Formar lectores y autores en un mundo visual. Cuadernos de Pedagogía, 363, 52-57.

Sancho, J. M. (10 de febrero 2017). No pongas tus ... manos sobre la educación. El Diario de la Educación. Recuperado de: eldiariodelaeducacion.com/blog/2017/02/10/ no-pongas-tus-manos-sobre-la-educacion/

Sancho, J. M. y Alonso, C. (Coord.) (2012). La fugacidad de las políticas, la inercia de las prácticas. Barcelona: Octaedro.

Sancho, J. M., Bosco, A., Alonso, C. y Sánchez, J. A. (2015). Formación del profesorado en Tecnología Educativa: de cómo las realidades generan los mitos. Revista Latinoamericana de Tecnología Educativa, 14(1), 17-29. doi: 10.17398/1695288X.

Sancho, J.M., Bosco, A., Ornellas, A., Sánchez, J.A. y Alonso, C. (2008), La formación del profesorado en el uso educativo de las TIC: una aproximación desde la política educativa, Praxis Educativa, 12, 10-22.

Sancho, J. M., y Butzbach, M. (1985). Informática educativa y formación permanente del profesorado: Un proyecto en desarrollo en Cataluña. En A. Peiffer y J. Galván (Eds.) Ob. cit. (pp. 249-254).

Savater, F. (1998). La educación que queremos. Recuperado de http://www.javeriana.edu. $\mathrm{co} /$ decisiones/savater.PDF

Swelyn, N. (2013). Distrusting Educational Technology: Critical Questions for Changing Times. New York/London: Routledge. Taylor \& Francis Group. 
Swelyn, N. (2016). Is Technology Good for Education? Cambridge: Polity Press.

Tedesco, J. C. (1995). El nuevo pacto educativo. Madrid: Anaya.

Twenge J. (2017). IGen: Why Today's Super-Connected Kids Are Growing Up Less Rebellious, More Tolerant, Less Happy--and Completely Unprepared for Adulthood--and What That Means for the Rest of Us. New York: ATRIA Books.

Tyack, D. y Tobin, W. (1994). The 'grammar' of schooling: why has it been so hard to change, American Educational Research Journal, 31(3), 453-480.

Weiser, M. (1991). The Computer for the Twenty-First Century. Scientific American, 265(3), 94-104.

\section{Agradecimientos}

ESBRINA- Subjetividades, visualidades y Entornos Educativos Contemporáneos - 2017SGR 1248: http://esbrina.eu

REUNI+D - Red Universitaria de Investigación e Innovación Educativa. Cambios sociales y retos para la educación. MINECO. EDU201568718-REDT: http://reunid.eu 